\title{
Engineering Note
}

ENGINEERING NOTES are short manuscripts describing new developments or important results of a preliminary nature. These Notes should not exceed 2500 words (where a figure or table counts as 200 words). Following informal review by the Editors, they may be published within a few months of the date of receipt. Style requirements are the same as for regular contributions (see inside back cover).

\section{Collisionless Gas Expanding into Vacuum}

\author{
Chunpei Cai* \\ ZONA Technology Inc., Scottsdale, Arizona 85258 \\ and \\ Iain D. Boydı \\ University of Michigan, Ann Arbor, Michigan 48109
}

DOI: $\underline{10.2514 / 1.32173}$

\begin{tabular}{|c|c|}
\hline & Nomenclature \\
\hline$f$ & $=$ velocity distribution function \\
\hline$n$ & $=$ number density \\
\hline$R$ & $\begin{array}{l}=\text { radius for a circular or an annular exit, universal gas } \\
\text { constant }\end{array}$ \\
\hline$r, \theta$ & $=$ polar coordinate variables \\
\hline$U, V, W$ & $=$ macroscopic average velocity \\
\hline$u, v, w$ & $=$ microscopic molecular velocity \\
\hline$X, Y, Z$ & $=\mathrm{a}$ point in front of the exit \\
\hline$y, z$ & $=\mathrm{a}$ point on the exit \\
\hline$\alpha$ & $\begin{aligned}= & \text { angle between the } X \text { axis and a segment from }(0,0) \\
& \text { to }(X, 0, Z)\end{aligned}$ \\
\hline$\beta$ & $=1 /\left(2 R T_{0}\right)$ \\
\hline
\end{tabular}

Subscript

$0=$ averaged property at the exit

\section{Introduction}

$\mathbf{H}$ IGH-SPEED collisionless, or free-molecular, gas flows passing through small circular or annular holes are fundamental problems with many real applications such as neutral gas expansion out of electric propulsion (EP) devices. Usually, the cold plume flow out of an EP device is modeled by assuming freemolecular flows with a nonzero uniform average exit velocity $U_{0}$. Even when the average bulk velocity of gas near the orifice is zero, the average velocity at the orifice exit plane is not zero, it corresponds to an outflow with a half-Maxwellian distribution. In the past, analytical studies of similar problems were concentrated on true effusion problems with a zero average exit speed. For example, Liepmann [1] reported the efflux of gases through circular apertures, which is an example of a transition from the gas-dynamic to the gaskinetic regime; Narasimha [2] obtained the exact solutions of density

\footnotetext{
Received 15 May 2007; revision received 24 July 2007; accepted for publication 26 July 2007. Copyright (C) 2007 by Chunpei Cai. Published by the American Institute of Aeronautics and Astronautics, Inc., with permission. Copies of this paper may be made for personal or internal use, on condition that the copier pay the $\$ 10.00$ per-copy fee to the Copyright Clearance Center, Inc., 222 Rosewood Drive, Danvers, MA 01923; include the code 0022-4650/07 \$10.00 in correspondence with the CCC.

*Computational Fluid Dynamics Specialist, 8489 East Ironwood Square Drive. Senior Member AIAA.

$\dagger$ Professor, Department of Aerospace Engineering, 1320 Beal Avenue. Associate Fellow AIAA.
}

and velocity distributions for a free-molecular effusion flow and the results for a nearly free-molecular effusion flow expanding into vacuum through a circular orifice; and Brook [3] reported the density field of free-molecular flow from an annulus, to study the gas leakage effect from a spacecraft hatch. Other researchers reported many approximate methods or numerical simulations to study rarefied flows through a slit; for example, Rotenberg and Weitzner [4], Hasegawa and Sone [5], Cercignani and Sharipov [6], and Sharipov [7]. Recently, Lilly et al. [8] reported their work on measurement and computation of mass flow and momentum flux through short tubes in rarefied gas. For the case of free-molecular flows with a nonzero average velocity, the problems are usually very complicated and approximations are often made, such as neglecting the details of the exit geometry or assuming that free-molecular flow are emitted from a point source [9].

In our previous study $[10,11]$, we adopted a relation between velocity directions and geometry locations to investigate freemolecular plume flow problems. This treatment is more general than the solid angle treatment [2]. which was widely used in studying true collisionless effusion flows with a zero average exit speed, but is not applicable to collisionless flows with a nonzero average exit speed.

In this study, we further investigate collisionless flows out of a circular or an annular exit with a nonzero average speed. These two cases are very important, not only because of their mathematical significance, but also because of their many direct applications, including spacecraft propulsion.

This Note is organized as follows: Section II describes the problems, the corresponding complex exact solutions, and also approximate far-field solutions, which are simpler and more accurate than existing formulas in the literature; Sec. III compares the analytical results with particle simulation results; and Sec. IV summarizes this study.

\section{Flow Problems and Solutions}

\section{A. Free-Molecular Problems}

The problems considered in this study are the following freemolecular gas flows expanding into vacuum from an exit: 1) a circular exit with a radius of $R$ and an average exit velocity $U_{0}$ that is greater than zero and 2) an annular exit characterized by an inner radius $R_{1}$, an outer radius $R_{2}$, and an average exit velocity $U_{0}$ that is greater than zero.

These two problems are closely related: if $R_{1}$ in the second problem is set to zero, then it degenerates to the first problem. Because both problems have important applications, we discuss each of them and provide the complete solutions.

The thermal velocity at the exits can be expressed with a Maxwellian distribution function characterized by a number density $n_{0}$ and a temperature $T_{0}$ :

$$
f(u, v, w)=n_{0}\left(\frac{\beta}{\pi}\right)^{3 / 2} \exp \left[-\beta\left(u^{2}+v^{2}+w^{2}\right)\right]
$$

where $\beta=1 / 2 R T_{0}$. Although the plume itself is in a highly nonequilibrium state, it is reasonable to assume that the flow is at equilibrium before it escapes from the exit. Using $T_{0}$ and $n_{0}$ to describe this equilibrium state is a natural selection, widely used by many researchers in the past [12]. 
This study adopts the following coordinate systems: Denote the plume direction as the $X$-axis direction, the direction normal to the $X$ axis as the $Z$-axis direction, and the circle center/annulus center as the origin. The objective of this study is to obtain the analytical plume field flow solutions, especially the number density and velocities at any point downstream of the exits.

\section{B. Collisionless Effusion Flow Solutions}

Narasimha [2] adopted solid angles to study a free-molecular flow of gas escaping from an orifice; however, this solid angle approach is not general enough to study effusion flows with nonzero average exit velocities. Instead, a relation of velocity directions and geometry positions was recently proposed [10].

For the circular exit case with a nonzero average exit speed, the relation between velocity direction and geometry position takes a new format. Suppose the average velocity at the circular exit is $U_{0}$, and from any point $(0, y, z)$ on the circular exit that is characterized by a radius of $R$, only particles with the following special velocity components can arrive at a point $(X, 0, Z)$ in front of the exit:

$$
\frac{X}{u+U_{0}}=\frac{Y-y}{v}=\frac{Z-z}{w}
$$

where $X>0, Y=0$, and $Z>0$. Combined with the geometry relations

$$
z=r \sin \theta=Z-\frac{X w}{u+U_{0}}, \quad y=r \cos \theta=Y-\frac{X v}{u+U_{0}}
$$

where $r \in[0, R]$ and $\theta \in[0,2 \pi]$, the integrals for the number density and the velocities can be simplified using the following change of variables:

$$
\mathrm{d} v \mathrm{~d} w=\left|\begin{array}{ll}
\frac{\partial v}{\partial r} & \frac{\partial v}{\partial \theta} \\
\frac{\partial w}{\partial r} & \frac{\partial w}{\partial \theta}
\end{array}\right| \mathrm{d} r \mathrm{~d} \theta=\frac{\left(u+U_{0}\right)^{2}}{X^{2}} r \mathrm{~d} r \mathrm{~d} \theta
$$

The preceding change of integral variables transfers the integral domains from $-\infty$ and $+\infty$ for $v$ and $w$ to finite spans for $r$ and $\theta$.

The final results of number density and velocities at a point $(X, 0, Z)$ in front of the exit are

$$
\begin{aligned}
& n(X, 0, Z)=\int_{-U_{0}}^{+\infty} \mathrm{d} u \int_{-\infty}^{+\infty} \mathrm{d} v \int_{-\infty}^{+\infty} \mathrm{d} w\left(\frac{\beta}{\pi}\right)^{3 / 2} \\
& \times \exp \left[-\beta\left(u^{2}+v^{2}+w^{2}\right)\right]=2\left(\frac{\beta}{\pi}\right)^{3 / 2} \int_{0}^{\infty} \mathrm{d} t \frac{t^{2}}{X^{2}} \int_{-\pi / 2}^{\pi / 2} \mathrm{~d} \theta \int_{0}^{R} r \mathrm{~d} r \\
& \times \exp \left[-\beta\left(t-U_{0}\right)^{2}-\beta \frac{t^{2}}{X^{2}}\left(r^{2}+Z^{2}-2 r Z \sin \theta\right)\right] \\
& =\frac{1}{X^{2}}\left(\frac{\beta}{\pi}\right)^{3 / 2} \int_{-\pi / 2}^{\pi / 2} \mathrm{~d} \theta \int_{0}^{R} \\
& \times \exp \left(-\beta U_{0}^{2} \frac{r^{2}+Z^{2}-2 Z r \sin \theta}{X^{2}+r^{2}+Z^{2}-2 Z r \sin \theta}\right) r K \mathrm{~d} r \\
& U(X, 0, Z)=\frac{1}{n(X, 0, Z) X^{2}}\left[\left(\frac{\beta}{\pi}\right)^{3 / 2} \int_{-\pi / 2}^{\pi / 2} \mathrm{~d} \theta \int_{0}^{R}\right. \\
& \left.\quad \times \exp \left(-\beta U_{0}^{2} \frac{r^{2}+Z^{2}-2 Z r \sin \theta}{X^{2}+r^{2}+Z^{2}-2 Z r \sin \theta}\right) M r \mathrm{~d} r\right] \\
& W(X, 0, Z)=\frac{1}{n(X, 0, Z) X^{2}}\left[\left(\frac{\beta}{\pi}\right)^{3 / 2} \int_{-\pi / 2}^{\pi / 2} \mathrm{~d} \theta \int_{0}^{R} \frac{Z^{2}-r \sin \theta}{X}\right. \\
& \left.\quad \exp \left(-\beta U_{0}^{2} \frac{r^{2}+Z^{2}-2 Z r \sin \theta}{X^{2}+r^{2}+Z^{2}-2 Z r \sin \theta}\right) M r \mathrm{~d} r\right]
\end{aligned}
$$

$$
\begin{aligned}
K & =\frac{Q^{2} U_{0}}{\beta} \exp \left(-\beta U_{0}^{2} Q\right)+\left(\frac{Q}{2 \beta}+Q^{2} U_{0}^{2}\right) \sqrt{\frac{\pi Q}{\beta}}[1 \\
& \left.+\operatorname{erf}\left(\sqrt{\beta Q} U_{0}\right)\right]
\end{aligned}
$$

$$
\begin{gathered}
Q=\frac{X^{2}}{X^{2}+Z^{2}+r^{2}-2 Z r \sin \theta} \\
M=\frac{Q^{3} U_{0}^{2}}{\beta} \exp \left(-\beta Q U_{0}^{2}\right)+\frac{Q^{2}}{\beta^{2}} \exp \left(-\beta Q U_{0}^{2}\right) \\
+\left(\frac{3 Q^{2} U_{0}}{2 \beta}+Q^{3} U_{0}^{3}\right) \sqrt{\frac{\pi Q}{\beta}}\left[1+\operatorname{erf}\left(\sqrt{\beta Q} U_{0}\right)\right]
\end{gathered}
$$

Similarly, the results for the annular case are very convenient to obtain by replacing the integral range for the exit radius from $0<r<R$ in Eqs. (5-7) by $R_{1}<r<R_{2}$.

\section{Far-Field Approximations}

Although the preceding exact relations are accurate and convenient to evaluate via a computer because the integration over the infinite span of velocity space is complete, they are rather complex for practical usage. Hence, approximate far-field simplifications are developed. Here, we provide far-field simplifications for the annular exit case, because the circular exit case can be obtained by setting $R_{1}=0$ from the following formula. From the preceding relations, with the far-field approximation $R_{1}<r<R_{2} \ll \sqrt{Z^{2}+X^{2}}, Q=\left[X^{2} /\left(X^{2}+Z^{2}\right)\right]=\cos ^{2} \alpha$,

$$
\begin{aligned}
& n(X, 0, Z)=\frac{R_{2}^{2}-R_{1}^{2}}{X^{2}} \cos ^{3} \alpha \\
& \quad \times\left[1-\frac{\beta U_{0}^{2}}{X^{2}+Z^{2}}\left(\frac{R_{1}^{2}+R_{2}^{2}}{2}+Z^{2}\right)\right] \\
& \quad \times\left(\frac{U_{0}}{2} \cos \alpha \sqrt{\frac{\beta}{\pi}} \exp \left(-\beta U_{0}^{2} \cos ^{2} \alpha\right)+\left(\frac{1}{4}+\frac{\beta \cos ^{2} \alpha U_{0}^{2}}{2}\right)\right. \\
& \left.\quad \times\left[1+\operatorname{erf}\left(\sqrt{\beta} \cos \alpha U_{0}\right)\right]\right)
\end{aligned}
$$

$$
\begin{aligned}
& U(X, 0, Z)=\frac{1}{n(X, 0, Z)} \sqrt{\frac{\beta}{\pi}} \frac{R_{2}^{2}-R_{1}^{2}}{2 X^{2}} \cos ^{4} \alpha \\
& \quad \times\left[1-\frac{\beta U_{0}^{2}}{X^{2}+Z^{2}}\left(\frac{R_{1}^{2}+R_{2}^{2}}{2}+Z^{2}\right)\right] \\
& \quad \times\left[\left(\cos ^{2} \alpha U_{0}^{2}+\frac{1}{\beta}\right) \exp \left(-\beta \cos ^{2} \alpha U_{0}^{2}\right)\right. \\
& \left.\quad+\left(\frac{3 \cos \alpha U_{0}}{2}+\beta U_{0}^{3} \cos ^{3} \alpha\right) \sqrt{\frac{\pi}{\beta}}\left[1+\operatorname{erf}\left(\sqrt{\beta} \cos \alpha U_{0}\right)\right]\right]
\end{aligned}
$$

$$
\begin{aligned}
& W(X, 0, Z)=\frac{1}{n(X, 0, Z)} \sqrt{\frac{\beta}{\pi}} \frac{R_{2}^{2}-R_{1}^{2}}{2 X^{2}} \cos ^{4} \alpha \\
& \quad \times\left[\frac{Z}{X}-\frac{\beta U_{0}^{2} Z}{X\left(X^{2}+Z^{2}\right)}\left(\frac{R_{2}^{2}+R_{1}^{2}}{2}+Z^{2}\right)-\frac{\left(R_{2}^{2}+R_{1}^{2}\right) Z}{2 X\left(X^{2}+Z^{2}\right)}\right] \\
& \quad \times\left[\left(\cos ^{2} \alpha U_{0}^{2}+\frac{1}{\beta}\right) \exp \left(-\beta \cos ^{2} \alpha U_{0}^{2}\right)\right. \\
& \left.\quad+\left(\frac{3 \cos \alpha U_{0}}{2}+\beta U_{0}^{3} \cos ^{3} \alpha\right) \sqrt{\frac{\pi}{\beta}}\left[1+\operatorname{erf}\left(\sqrt{\beta} \cos \alpha U_{0}\right)\right]\right]
\end{aligned}
$$


The cosine function dominates in the far-field simplifications, and by retaining the geometry radius in the expressions, these relations are more accurate than the results obtained by Narashima [9]. It is also possible to estimate how far from the exit these approximations are accurate. By comparison, the point source solutions [9] totally neglect the exit geometry, hence their accuracy should be inferior to the preceding approximations.

\section{Centerline Property Distributions}

The centerline property distributions can be obtained from the exact solutions by setting $Z=0$. The final solutions to the circular exit case are

$$
\begin{aligned}
& n(X, 0,0)=\frac{1}{2}+\frac{1}{2} \operatorname{erf}\left(\sqrt{\beta} U_{0}\right)-\frac{X}{2 \sqrt{X^{2}+R^{2}}} \exp \left(-\frac{\beta R^{2} U_{0}^{2}}{X^{2}+R^{2}}\right) \\
& \times\left[1+\operatorname{erf}\left(\sqrt{\beta \frac{X^{2}}{X^{2}+R^{2}}} U_{0}\right)\right] \\
& U(X, 0,0)=\frac{1}{2 n(X, 0,0)}\left\{\frac{R^{2} \exp \left(-\beta U_{0}^{2}\right)}{\sqrt{\beta \pi}\left(X^{2}+R^{2}\right)}+U_{0}\left[1+\operatorname{erf}\left(\sqrt{\beta} U_{0}\right)\right]\right. \\
& -\exp \left(-\frac{\beta R^{2} U_{0}^{2}}{X^{2}+R^{2}}\right) \frac{U_{0} X^{3}}{\left(X^{2}+R^{2}\right)^{3 / 2}} \\
& \left.\quad \times\left[1+\operatorname{erf}\left(\sqrt{\beta \frac{X^{2}}{X^{2}+R^{2}}} U_{0}\right)\right]\right\}
\end{aligned}
$$

The corresponding solutions to the annular exit case are

$$
\begin{aligned}
& n(X, 0,0)=\frac{X}{2 \sqrt{X^{2}+R_{1}^{2}}} \exp \left(-\frac{\beta R_{1}^{2} U_{0}^{2}}{X^{2}+R_{1}^{2}}\right) \\
& \times\left[1+\operatorname{erf}\left(\sqrt{\beta \frac{X^{2}}{X^{2}+R_{1}^{2}}} U_{0}\right)\right]-\frac{X}{2 \sqrt{X^{2}+R_{2}^{2}}} \exp \left(-\frac{\beta R_{2}^{2} U_{0}^{2}}{X^{2}+R_{2}^{2}}\right) \\
& \times\left[1+\operatorname{erf}\left(\sqrt{\beta \frac{X^{2}}{X^{2}+R_{2}^{2}}} U_{0}\right)\right] \\
& U(X, 0,0)=\frac{1}{2 n(X, 0,0)}\left\{\frac{X^{2}}{\sqrt{\beta \pi}}\left(\frac{1}{X^{2}+R_{1}^{2}}-\frac{1}{X^{2}+R_{2}^{2}}\right)\right. \\
& \times \exp \left(-\beta U_{0}^{2}\right)+\exp \left(-\frac{\beta R_{1}^{2}}{X^{2}+R_{1}^{2}}\right) \frac{U_{0} X^{3}}{\left(X^{2}+R_{1}^{2}\right)^{3 / 2}} \\
& \times\left[1+\operatorname{erf}\left(\sqrt{\beta \frac{X^{2}}{X^{2}+R_{1}^{2}}} U_{0}\right)\right]-\exp \left(-\frac{\beta R_{2}^{2} U_{0}^{2}}{X^{2}+R_{2}^{2}}\right) \frac{U_{0} X^{3}}{\left(X^{2}+R_{2}^{2}\right)^{3 / 2}} \\
& \left.\times\left[1+\operatorname{erf}\left(\sqrt{\beta \frac{X^{2}}{X^{2}+R_{2}^{2}}} U_{0}\right)\right]\right\}
\end{aligned}
$$

It is worthy to mention that to the authors' knowledge, there were no previous results for this problem that include the geometry factors. If $U_{0}=0$, the centerline results degenerate to those for effusion flow problems [11].

\section{Simulations and Discussion}

Although the complete analytical results involve several integral terms that cannot be explicitly removed, numerical evaluations are convenient via a computer. The subroutine for the error function can be found in many numerical computing books [13]. Because the flows are rarefied, it is appropriate to use the direct simulation Monte Carlo (DSMC) method [14] to validate the analytical results. In this study, we used a specific DSMC package named MONACO [15] to perform the simulations. The simulation domain and mesh are quite simple, and the collision function in MONACO is turned off to

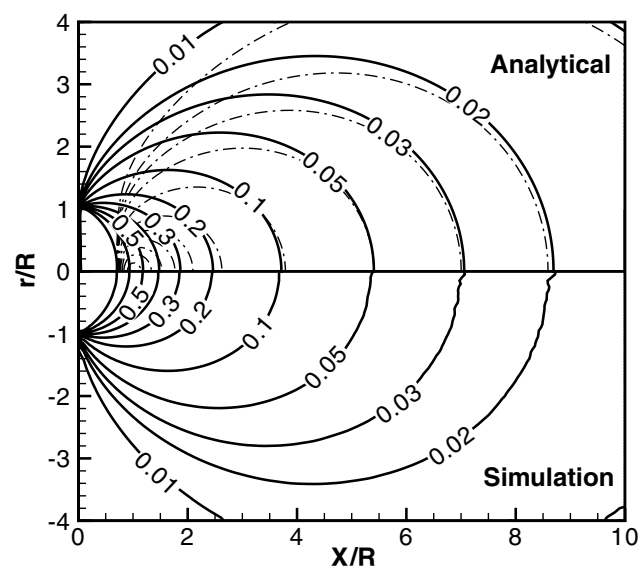

Fig. 1 Contours of $n(X, 0, Z) / n_{0}$ (circular exit: $U_{0}=\sqrt{2 R T_{0}}$; dashed line: analytical far-field approximations).

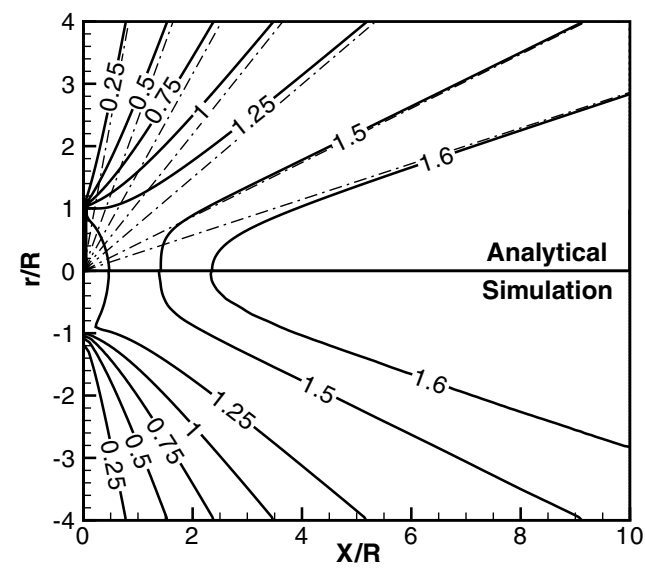

Fig. 2 Contours of $U(X, 0, Z) / \sqrt{2 R T_{0}}$ (circular exit: $U_{0}=\sqrt{2 R T_{0}}$; dashed line: analytical far-field approximations).

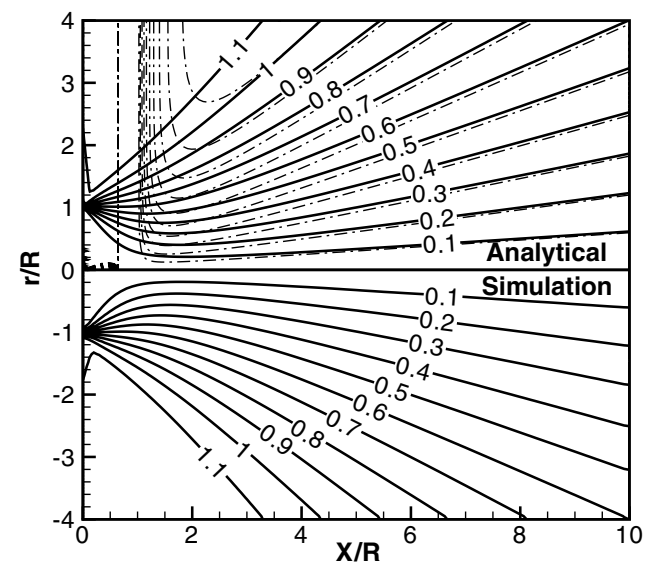

Fig. 3 Contours of $W(X, 0, Z) / \sqrt{2 R T_{0}}$ (circular exit: $U_{0}=\sqrt{2 R T_{0}}$; dashed line: analytical far-field approximations).

achieve the collisionless effect. Under this situation, the value of the number density at the exit does not produce any difference in the final normalized results, and exact free-molecular flows are guaranteed.

Figure 1 shows comparisons of number density contours from the exact analytical solutions, the far-field approximations, and the DSMC results. On the top, the contours with solid lines are the exact analytical solutions, and the contours with dashed lines are the farfield approximation results. The contours at the bottom are the DSMC simulation results. The average exit velocity at the slit is set to $\sqrt{2 R T_{0}}$ and the exit temperature is set to $T=300 \mathrm{~K}$. By turning off 


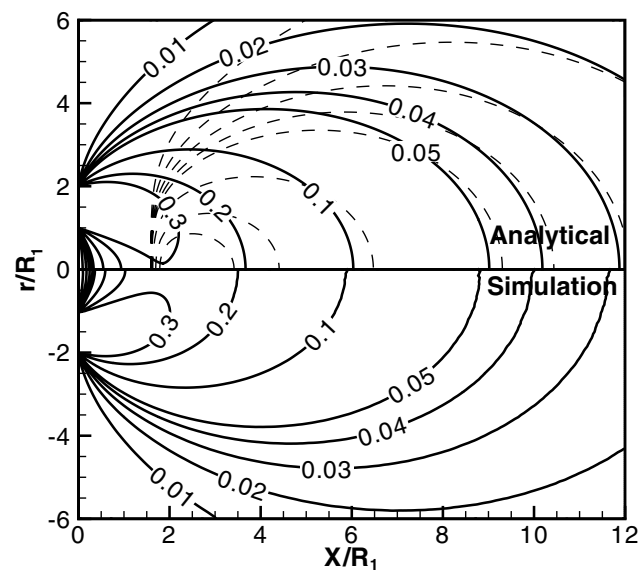

Fig. 4 Contours of $n(X, 0, Z) / n_{0}$ (annular exit: $R_{2}=2 R_{1}$ and $U_{0}=\sqrt{2 R T_{0}}$; dashed line: analytical far-field approximations).

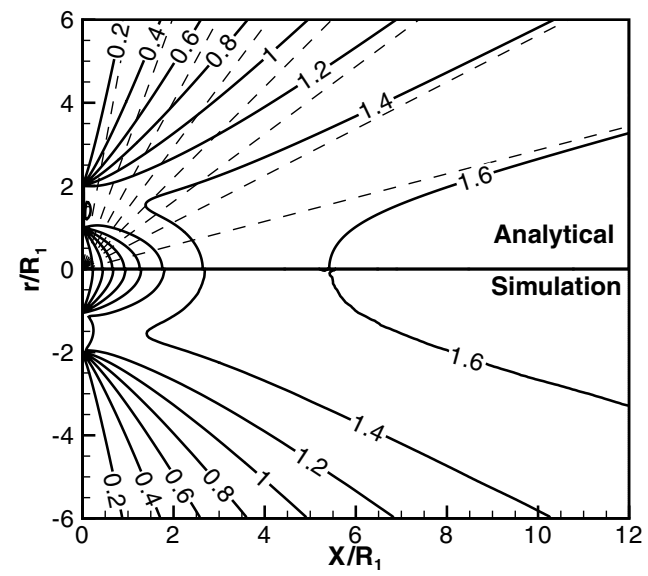

Fig. 5 Contours of $U(X, 0, Z) / \sqrt{2 R T_{0}}$ (annular exit: $R_{2}=2 R_{1}$ and $U_{0}=\sqrt{2 R T_{0}}$; dashed line: analytical far-field approximations).

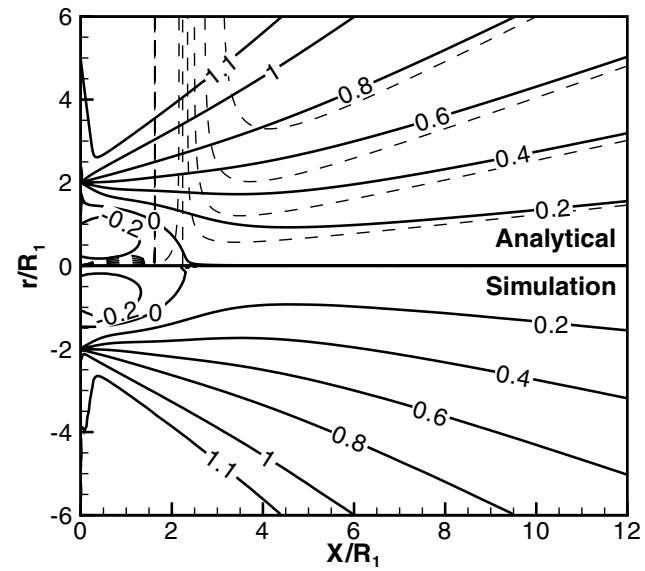

Fig. 6 Contours of $W(X, 0, Z) / \sqrt{2 R T_{0}}$ (annular exit: $R_{2}=2 R_{1}$ and $U_{0}=\sqrt{2 R T_{0}}$; dashed line: analytical far-field approximations).

the collision function in the DSMC method, the flows are exactly free-molecular. Generally, the exact analytical results are almost identical to the simulation results. There are some minor differences at the near field close to the exit, because the numerical evaluation of the exact solutions contains very small denominator terms in these regions, especially close to the origin. It is also very clear from the plot that the far-field approximations are not very accurate when $X<3 R$, but the accuracy significantly improves when $X>3 R$. Figures $\underline{2}$ and $\underline{3}$ show the corresponding results of velocity contours normalized with the characterized thermal speed $\sqrt{2 R T_{0}}$.
Conclusions similar to the density distributions can be drawn from these two pictures. The flow patterns have a narrow zone in which exit effects dominate, but in the far field, the contour lines are straight.

We also perform a simulation to validate the analytical results for the annulus case. In this simulation, the inner and outer radii of the annulus are set to $R_{1}=0.1 \mathrm{~m}$ and $R_{2}=0.2 \mathrm{~m}$, respectively.

Figure 4 shows contours of normalized number density. In the whole simulation domain, the comparison shows very close results between the exact analytical solutions and the numerical simulations, and the far-field simplifications become very accurate when $X>3 R_{2}$. Figures $\underline{5}$ and $\underline{6}$ show the velocity contours. Both comparisons are very satisfactory as well. In all of these three pictures, both the exit region and the slow "cavity" region in the center, which is characterized by negative $W_{z}$, are clearly captured.

\section{Conclusions}

This study is a natural extension of the previous work [10] aiming to seek analytical results for free-molecular plume flows from EP devices designed for spacecraft propulsion. We reported analytical solutions to two fundamental free-molecular flows out of a circular or an annular exit with nonzero average speed and validations with particle simulations. More specifically,

1) The analytical results indicate that the solutions are composed of complex geometry factors and the average exit velocity $U_{0}$. Even though the formulas are complex, we evaluated the analytical results and compared them with the DSMC simulation results. The excellent match between the numerical results and the analytical results indicates that the treatment in this study was correct. The accuracy of the exact solutions provides a solid foundation for far-field approximations and centerline property distributions.

2) The far-field solutions of number density and velocities contain the exit geometry factors. Hence, they are more accurate than the point source solutions obtained by Narasimha [9]. Comparisons with numerical simulation results indicate that the far-field approximations are accurate when $X>3 R$ for a circular exit and $X>3 R_{2}$ for an annular exit.

\section{References}

[1] Liepmann, H. W., "Gas Kinetics and Gasdynamics of Orifice Flow," Journal of Fluid Mechanics, Vol. 10, No. 1, 1961, pp. 65-79. doi:10.1017/S002211206100007X

[2] Narasimha, R., "Orifice Flow of High Knudsen Number," Journal of Fluid Mechanics, Vol. 10, No. 3, 1961, pp. 371-384. doi:10.1017/S0022112061000986

[3] Brook, J. W., "Density Field of Directed Free-Molecular Flow from an Annulus," Journal of Spacecraft and Rockets, Vol. 6, No. 6, 1969, pp. 755-757.

[4] Rotenberg, A. N., and Weitzner, H., "Nearly Free Flow Through an Orifice," Physics of Fluids, Vol. 12, No. 8, 1969, pp. 1573-1581. doi:10.1063/1.1692713

[5] Hasegawa, M., and Sone, Y., "Rarefied Gas Flow Through a Slit," Physics of Fluids A, Vol. 3, No. 3, Mar. 1991, pp. 466-477. doi: $10.1063 / 1.858103$

[6] Cercignani, C., and Sharipov, F., "Gaseous Mixture Slit Flow at Intermediate Knudsen Numbers," Physics of Fluids A, Vol. 4, No. 6, June 1992, pp. 1283-1289. doi: $10.1063 / 1.858246$

[7] Sharipov, F., "Non-Isothermal Rarefied Gas Flow Through a Slit," Physics of Fluids, Vol. 9, No. 6, June 1997, pp. 1804-1810. doi:10.1063/1.869295

[8] Lilly, T. C., Gimelshein, S. F., Ketsdever, A. D., and Markelov, G. N., "Measurements and Computations of Mass Flow and Momentum Flux Through Short Tubes in Rarefied Gases," Physics of Fluids, Vol. 18, No. 9, 2006, p. 093601. doi: $10.1063 / 1.2345681$

[9] Narasimha, R., "Collisionless Expansion of Gases into Vacuum," Journal of Fluid Mechanics Vol. 12, No. 3, 1962, pp. 294-308. doi:10.1017/S0022112062000208

[10] Cai, C., and Boyd, I. D., "Theoretical and Numerical Study of Several Free Molecular Flow Problems" Journal of Spacecraft and Rockets, Vol. 44, No. 3, May-June 2007; also AIAA Paper 2006-3800, 2006.

[11] Cai, C., "Theoretical and Numerical Studies of Plume Flows in Vacuum 
Chambers," Ph.D. Dissertation, Dept. of Aerospace Engineering, Univ. of Michigan, Ann Arbor, Michigan, Oct. 2005.

[12] Gombosi, T. I., Gaskinetic Theory, Cambridge Univ. Press, New York, 1994.

[13] Press, W. H., Vetterling, S. A., Flannery, W. T., and Flannery, B. P., Numerical Recipes in C, Cambridge Univ. Press, New York, 1994.

[14] Bird, G. A., Molecular Gas Dynamics and the Direct Simulation of Gas Flows, Oxford Univ. Press, New York, 1994.
[15] Dietrich, S., and Boyd, I. D., "Scalar and Parallel Optimized Implementation of the Direct Simulation Monte Carlo Method," Journal of Computational Physics, Vol. 126, No. 2, 1996, pp. 328-342. doi:10.1006/jcph.1996.0141

A. Ketsdever Associate Editor 